\title{
Dynamic shell buckling behavior of multi-walled carbon nanotubes embedded in an elastic medium
}

\author{
SUN ChengQi ${ }^{1 *}$, LIU KaiXin $^{2} \&$ HONG YouShi ${ }^{1}$ \\ ${ }^{1}$ State Key Laboratory of Nonlinear Mechanics, Institute of Mechanics, Chinese Academy of Sciences, Beijing 100190, China; \\ ${ }^{2}$ LTCS and Department of Mechanics \& Aerospace Engineering, College of Engineering, Peking University, Beijing 100871, China
}

Receiced September 19, 2012; accepted December 14, 2012

\begin{abstract}
This paper studies the dynamic shell buckling behavior of multi-walled carbon nanotubes (MWNTs) embedded in an elastic medium under step axial load based on continuum mechanics model. It is shown that, for occurrence of dynamic shell buckling of MWNTs or MWNTs embedded in an elastic medium, the buckling stress is higher than the critical buckling stress of the corresponding static shell buckling under otherwise identical conditions. Detailed results are demonstrated for dynamic shell buckling of individual double-walled carbon nanotubes (DWNTs) or DWNTs embedded in an elastic medium. A phenomenon is shown that DWNTs or embedded DWNTs in dynamic shell buckling are prone to axisymmetric buckling rather than non-axisymmetric buckling. Numerical results also indicate that the axial buckling form shifts from the lower buckling mode to the higher buckling mode with increasing buckling stress, but the buckling mode is invariable for a certain domain of buckling stress. Further, an approximate analytic formula is presented for the buckling stress and the associated buckling wavelength for dynamic axisymmetric buckling of embedded DWNTs. The effect of radii is also examined.
\end{abstract}

dynamic buckling, carbon nanotube, van der Waals forces, buckling stress, buckling mode

PACS number(s): 61.46.Fg, 46.70.-p, 46.32.+x

Citation: Sun C Q, Liu K X, Hong Y S. Dynamic shell buckling behavior of multi-walled carbon nanotubes embedded in an elastic medium. Sci China-Phys Mech Astron, 2013, 56: 483-490, doi: 10.1007/s11433-013-5009-5

\section{Introduction}

Carbon nanotubes (CNTs) have excellent mechanical properties and a wide range of applications, such as structural elements for nano-electromechanical systems and nanocomposites [1,2]. Mechanical behaviors of CNTs or CNTs in various surroundings have been widely investigated by experiments, molecular dynamic simulations and continuum mechanics models [3-6]. For example, Yakobson et al. [7] studied the axial compressive buckling of SWNTs and found that the continuum shell model predicted well all the changes of buckling patterns displayed by molecular dynamics simulations. $\mathrm{Ru}[8,9]$ considered the effect of inter-

*Corresponding author (email: scq@1nm.imech.ac.cn) tube van der Waals interaction and proposed a double-shell model for studying the axial compressive buckling of DWNTs and DWNTs in an elastic medium. Wang et al. [10] studied the size dependence of thin shell model for CNTs and showed that the size dependence was insignificant for SWNTs with diameter bigger than $1.5 \mathrm{~nm}$. Some other researches have also indicated that the continuum mechanical models can present the major factor affecting the mechanical behavior of CNTs and can be effectively used to study the mechanical deformation of CNTs [11-13].

CNTs have potential importance in the use as basic elements of nanoscale devices such as chemical and mechanical sensors [2,14]. The reliability of many devices depends critically on the understanding of the responses of CNTs to external loadings, thus studying the dynamic buckling be- 
havior of MWNTs or MWNTs in an elastic medium is of great importance. In previous papers $[15,16]$, the dynamic torsional buckling behavior of MWNTs embedded in an elastic medium and dynamic column bucking behavior of MWNTs under step axial load have been studied by introducing initial imperfections for MWNTs and applying the preferred mode analytical method based on continuum mechanics model. MWNTs are hollow multilayer structure and originally concentric, so they may exhibit various buckling behaviors such as shell buckling, column buckling, torsional buckling or bending, because of their different aspect ratios and loading conditions.

In this paper, a study is performed for the dynamic shell buckling behavior of MWNTs embedded in an elastic medium under step axial load based on continuum mechanics model, which takes into account the effect of radial constraint from the surrounding elastic medium and the effect of van der Waals forces between adjacent tubes. A buckling condition is derived for the buckling stress and the associated buckling mode, and detailed results are demonstrated for dynamic shell buckling of individual DWNTs and DWNTs embedded in an elastic medium.

\section{Multiple-shell model}

\subsection{The van der Waals forces}

Figure 1 shows an MWNT of length $L$ embedded in an elastic medium. Subscripts $1,2, \ldots, N$ denote the corresponding quantities related to the innermost tube, its adjacent tube, ..., the outermost tube, respectively. The (inward) pressures $p_{k(k+1)}$ exerted on the $k^{\text {th }}$ tube due to the $(k+1)^{\text {th }}$ tube and the (inward) pressures $p_{(k+1) k}$ exerted on the $(k+1)^{\text {th }}$ tube due to the $k^{\text {th }}$ tube are related by $[9,11]$

$$
R_{k} p_{k(k+1)}=-R_{k+1} p_{(k+1) k},(k=1,2, \ldots, N-1),
$$

where $R_{k}$ is the radius of the $k^{\text {th }}$ tube.

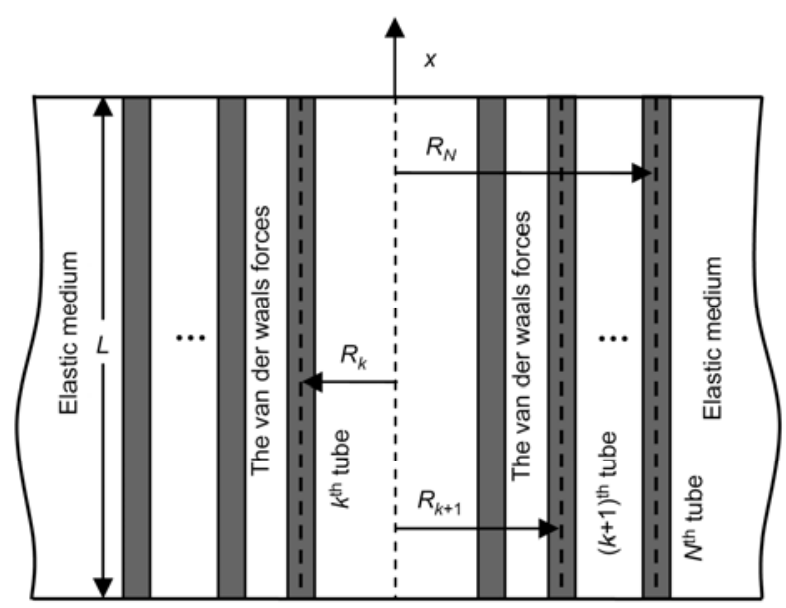

Figure 1 MWNT embedded in an elastic medium.
The pressures caused by van der Waals forces due to buckling are described as an approximate linear relation $[11,15]$ given as:

$$
\begin{aligned}
& p_{12}=c\left(w_{2}-w_{1}\right), \\
& p_{23}=c\left(w_{3}-w_{2}\right), \ldots, p_{(N-1) N}=c\left(w_{N}-w_{N-1}\right),
\end{aligned}
$$

where the van der Waals constant $c$ is estimated by

$$
c=\frac{320 \mathrm{erg} / \mathrm{cm}^{2}}{0.16 \mathrm{~s}^{2}}, s=0.142 \mathrm{~nm} .
$$

\subsection{The surrounding elastic medium}

During the buckling of an MWNT embedded in an elastic medium, an interaction pressure is usually induced between the outermost tube of the MWNT and the surrounding elastic medium. The pressure $p_{d}$ exerted on the outermost tube caused by the surrounding elastic medium $[4,9]$ is described as:

$$
p_{d}=-d w_{N}
$$

where $d$ is a spring constant determined by the material properties of both the elastic medium and the outermost tube of the MWNT.

In the present model, the Whitney-Riley model is used to determine the spring constant $d$. As that used by Wang et al. [4], the surrounding elastic medium is regarded as a hollow cylinder with inner radius $R_{N}$ and outer radius $r_{m}$, and the spring constant $d$ is determined as:

$$
d=\frac{E_{m}\left(r_{m}^{2}-R_{N}^{2}\right)}{\left(1+v_{m}\right) R_{N}\left[r_{m}^{2}+\left(1-2 v_{m}\right) R_{N}^{2}\right]},
$$

where $E_{m}$ is Young's modulus and $v_{m}$ is Poisson ratio of the surrounding elastic medium.

It can be seen from eq. (4) that, for an MWNT embedded in an infinite elastic medium $\left(r_{m} \rightarrow \infty\right)$, the spring constant $d$ is expressed as:

$$
d=\frac{E_{m}}{\left(1+v_{m}\right) R_{N}}
$$

\subsection{The governing buckling equation}

Consider a shell impacted by a step axial load at its end $(x=0)$. Denoting time by $t$ and the axial load per unit length by $N(t)=-N_{x} H(t)$, where $N_{x}$ is a constant, and the step function $H(t)=1$ if $t \geqslant 0 ; H(t)=0$ if $t<0$. Thus, the governing equation for dynamic buckling of an elastic shell is expressed as:

$$
D \nabla^{8} w=\nabla^{4}\left[p(x, \theta, t)-N_{x} \frac{\partial^{2} w}{\partial x^{2}}-\rho h \frac{\partial^{2} w}{\partial t^{2}}\right]-\frac{E h}{R^{2}} \frac{\partial^{4} w}{\partial x^{4}},
$$


ferential angular coordinate, $w(x, \theta, t)$ denotes the additional radial (inward) displacement of the middle surface due to buckling, $D$ and $h$ are the bending stiffness and thickness of the elastic shell, $E$ is Young's modulus, and $p(x, \theta, t)$ is the net (inward) normal pressure because of buckling.

It is assumed that each layer of an MWNT can be seen as an individual elastic shell and that the deflections of all layers are coupled through the van der Waals interaction between adjacent tubes. By use of eqs. (1)-(3) and (6), the governing equations for an embedded $N$-layer MWNT impacted by a step axial load at its end $(x=0)$ are obtained as:

$$
\begin{aligned}
D_{1} \nabla_{1}^{8} w_{1}= & \nabla_{1}^{4}\left[-N_{x 1} \frac{\partial^{2} w_{1}}{\partial x^{2}}-\rho h_{1} \frac{\partial^{2} w_{1}}{\partial t^{2}}+c\left(w_{2}-w_{1}\right)\right] \\
& -\frac{E h_{1}}{R_{1}^{2}} \frac{\partial^{4} w_{1}}{\partial x^{4}}, \\
D_{k} \nabla_{k}^{8} w_{k}= & \nabla_{k}^{4}\left[-N_{x k} \frac{\partial^{2} w_{k}}{\partial x^{2}}-\rho h_{k} \frac{\partial^{2} w_{k}}{\partial t^{2}}+c\left(w_{k+1}-w_{k}\right)\right. \\
& \left.-\frac{R_{k-1}}{R_{k}} c\left(w_{k}-w_{k-1}\right)\right]-\frac{E h_{k}}{R_{k}^{2}} \frac{\partial^{4} w_{k}}{\partial x^{4}} \\
& (k=2,3, \ldots, N-1), \\
D_{N} \nabla_{N}^{8} w_{N}= & \nabla_{N}^{4}\left[-N_{x N} \frac{\partial^{2} w_{N}}{\partial x^{2}}-\rho h_{N} \frac{\partial^{2} w_{N}}{\partial t^{2}}\right. \\
& \left.-c \frac{R_{N-1}}{R_{N}}\left(w_{N}-w_{N-1}\right)-d w_{N}\right]-\frac{E h_{N}}{R_{N}^{2}} \frac{\partial^{4} w_{N}}{\partial x^{4}},
\end{aligned}
$$

where $w_{k}(x, \theta, t)(k=1,2, \ldots, N)$ is the (inward) deflection of the $k^{\text {th }}$ tube, $D_{k}$ and $h_{k}$ are the effective bending stiffness and thickness of the $k^{\text {th }}$ tube, $\nabla_{k}^{2}=\frac{\partial^{2}}{\partial x^{2}}+\frac{1}{R_{k}^{2}} \frac{\partial^{2}}{\partial \theta^{2}}, k=1,2, \ldots$, $N$.

In this paper, we take $D_{k}=D=0.85 \mathrm{eV}, E h_{k}=E h=360 \mathrm{~J} / \mathrm{m}^{2}$, $\rho h_{k}=\rho h=\left(2.27 \mathrm{~g} / \mathrm{cm}^{3}\right) \times 0.34 \mathrm{~nm}[11,12,15], k=1,2, \ldots, N$, $E_{m}=2.03 \mathrm{GPa}$ and $v_{m}=0.33$ for the surrounding elastic medium $[4,17]$.

\section{Buckling analysis of embedded MWNTs}

The simply supported boundary conditions are considered for MWNTs embedded in an elastic medium, and the deflection of the $k^{\text {th }}$ layer can be expressed as:

$$
w_{k}(x, \theta, t)=\sum_{m=1}^{\infty} \sum_{n=0}^{\infty} f_{k}^{m n}(x, \theta) T_{m n}(t),(k=1,2, \ldots, N) .
$$

The initial conditions satisfy

$$
w_{k}(x, \theta, 0)=w_{k}^{0} \text { and } \partial w_{k}(x, \theta, 0) / \partial t=0(k=1,2, \ldots, N),(9)
$$

where $w_{k}^{0}(k=1,2, \ldots, N)$ denotes the initial imperfections prior to buckling for the $k^{\text {th }}$ layer of embedded MWNTs, and can be expanded as:

$$
w_{k}^{0}=\sum_{m=1}^{\infty} \sum_{n=0}^{\infty} A_{k}^{m n} \sin \frac{m \pi x}{L} \cos (n \theta)(k=1,2, \ldots, N),
$$

where $A_{k}^{m n}(k=1,2, \ldots, N)$ are real constants, $m$ and $n$ represent the half wave numbers in axial and circumferential directions, respectively.

Substituting eq. (8) into eq. (7) and using separation of variables leads to

$$
-\frac{1}{T_{m n}(t)} \frac{\partial^{2} T_{m n}(t)}{\partial t^{2}}=-\omega^{\prime 2}
$$

and

$$
\begin{aligned}
& D_{1} \nabla_{1}^{8} f_{1}^{m n}-\nabla_{1}^{4}\left[-\sigma_{x} h \frac{\partial^{2} f_{1}^{m n}}{\partial x^{2}}+c\left(f_{2}^{m n}-f_{1}^{m n}\right)-\rho h \omega^{\prime 2} f_{1}^{m n}\right] \\
& +\frac{E h}{R_{1}^{2}} \frac{\partial^{4} f_{1}^{m n}}{\partial x^{4}}=0 \\
& D_{k} \nabla_{k}^{8} f_{k}^{m n}-\nabla_{k}^{4}\left[-\sigma_{x} h \frac{\partial^{2} f_{k}^{m n}}{\partial x^{2}}+c\left(f_{k+1}^{m n}-f_{k}^{m n}\right)\right. \\
& \left.-\frac{R_{k-1}}{R_{k}} c\left(f_{k}^{m n}-f_{k-1}^{m n}\right)-\rho h \omega^{\prime 2} f_{k}^{m n}\right] \\
& +\frac{E h}{R_{k}^{2}} \frac{\partial^{4} f_{k}^{m n}}{\partial x^{4}}=0(k=2,3, \ldots, N-1) \\
& D_{N} \nabla_{N}^{8} f_{N}^{m n}-\nabla_{N}^{4}\left[-\sigma_{x} h \frac{\partial^{2} f_{N}^{m n}}{\partial x^{2}}-c \frac{R_{N-1}}{R_{N}}\left(f_{N}^{m n}-f_{N-1}^{m n}\right)\right. \\
& \left.-d f_{N}^{m n}-\rho h \omega^{\prime 2} f_{N}^{m n}\right]+\frac{E h}{R_{N}^{2}} \frac{\partial^{4} f_{N}^{m n}}{\partial x^{4}}=0
\end{aligned}
$$

where $\sigma_{x}$ denotes the applied axial stress.

From eq. (11), we have

$$
T_{m n}(t)=\left\{\begin{array}{l}
b_{m n} e^{\omega t}+b_{m n}^{\prime} e^{-\omega t}, \omega^{\prime 2}>0, \\
b_{m n}+b_{m n}^{\prime} t, \omega^{\prime 2}=0, \\
b_{m n} \sin (\omega t)+b_{m n}^{\prime} \cos (\omega t), \omega^{\prime 2}<0,
\end{array}\right.
$$

where $\omega(m, n)=\left|\omega^{\prime}(m, n)\right|$.

Eq. (13) corresponds to vibrational form of embedded MWNTs when $\omega^{\prime 2}<0$ and static buckling behavior of embedded MWNTs when $\omega^{\prime 2}=0$ [9]. Therefore, for dynamic shell buckling behavior of embedded MWNTs, $\omega^{\prime 2}>0$.

By virtue of the initial conditions of eq. (10), the buckling deflections of an embedded MWNT can be expressed as:

$$
w_{k}=\sum_{m=1}^{\infty} \sum_{n=0}^{\infty} A_{k}^{m n} \sin \frac{m \pi x}{L} \cos (n \theta) g(\omega, t) \quad(k=1,2, \ldots, N),
$$


where $g(\omega, t)=\cosh (\omega t)=\left(\mathrm{e}^{\omega t}+\mathrm{e}^{-\omega t}\right) / 2$ is amplification function.

Therefore, a set of $N$ homogeneous equations on $A_{k}^{m n}(k$ $=1,2, \ldots, N$ ) are obtained from eq. (12)

$$
\begin{gathered}
M_{11}^{m n} A_{1}^{m n}+M_{12}^{m n} A_{2}^{m n}=0, \\
M_{k(k-1)}^{m n} A_{k-1}^{m n}+M_{k k}^{m n} A_{k}^{m n}+M_{k(k+1)}^{m n} A_{k+1}^{m n}=0 \\
(k=2,3, \ldots, N-1),
\end{gathered}
$$

$$
M_{N(N-1)}^{m n} A_{N-1}^{m n}+M_{N N}^{m n} A_{N}^{m n}=0,
$$

where

$$
\begin{aligned}
& M_{11}^{m n}=D_{1} \beta_{1}^{4}+\left(c-\sigma_{x} h \frac{m^{2} \pi^{2}}{L^{2}}+\rho h \omega^{2}\right) \beta_{1}^{2}+\frac{m^{4} \pi^{4}}{L^{4}} \frac{E h}{R_{1}^{2}}, \\
& M_{12}^{m n}=-c \beta_{1}^{2}, \\
& M_{k(k-1)}^{m n}=-c \frac{R_{k-1}}{R_{k}} \beta_{k}^{2}, \\
& M_{k k}^{m n}=D_{k} \beta_{k}^{4}+\left(c \frac{R_{k-1}}{R_{k}}+c-\sigma_{x} h \frac{m^{2} \pi^{2}}{L^{2}}+\rho h \omega^{2}\right) \beta_{k}^{2} \\
& \quad+\frac{m^{4} \pi^{4}}{L^{4}} \frac{E h}{R_{k}^{2}}, \\
& M_{k(k+1)}^{m n}=-c \beta_{k}^{2}, \\
& M_{N(N-1)}^{m n}=-c \frac{R_{N-1}}{R_{N}} \beta_{N}^{2}, \\
& M_{N N}^{m n}=D_{N} \beta_{N}^{4}+\left(c \frac{R_{N-1}}{R_{N}}+d-\sigma_{x} h \frac{m^{2} \pi^{2}}{L^{2}}+\rho h \omega^{2}\right) \beta_{N}^{2} \\
& \quad+\frac{m^{4} \pi^{4}}{L^{4}} \frac{E h}{R_{N}^{2}}, \\
& \beta_{k}=\frac{m^{2} \pi^{2}}{L^{2}}+\frac{n^{2}}{R_{k}^{2}}, \quad(k=1,2, \ldots, N) .
\end{aligned}
$$

Eq. (15) can be written as:

$$
\boldsymbol{M}^{m n} \cdot\left[A_{1}^{m n}, A_{2}^{m n}, \ldots, A_{N}^{m n}\right]^{\mathrm{T}}=0,
$$

where $\boldsymbol{M}^{m n}$ denotes the coefficient matrix on $A_{1}^{m n}$, $A_{2}^{m n}, \ldots, A_{N}^{m n}$.

The requirement that eq. (16) has a non-zero solution on $A_{k}^{m n}(k=1,2, \ldots, N)$ leads to

$$
\operatorname{det} \boldsymbol{M}^{m n}=0 .
$$

From eq. (17), the equation on $-\sigma_{x} h m^{2} \pi^{2} L^{-2}+\rho h \omega^{2}$ is obtained, which shows the relation between applied axial stress $\sigma_{x}, \omega$ and the associated buckling mode $(m, n)$. From this equation, it is seen that the value of $\sigma_{x}$ increases monotonously with $\omega \geqslant 0$ for a given buckling mode $(m, n)$. This indicates that, for occurrence of dynamic shell buckling of
MWNTs embedded in an elastic medium, the buckling stress $\sigma_{x}$ is higher than the corresponding static critical buckling stress $\sigma_{c r}$ under otherwise identical conditions. Therefore, for the applied axial stress $\sigma_{x}$ larger than $\sigma_{c r}$, a set of discrete values of $\omega\left(0<\omega_{m_{1} n_{1}}<\omega_{m_{2} n_{2}}<\ldots<\omega_{m_{M} n_{M}}\right)$ are obtained, and the associated buckling modes $\left(m_{1}, n_{1}\right)$, $\left(m_{2}, n_{2}\right), \ldots,\left(m_{M}, n_{M}\right)$ are determined. From eq. (14), the deflections of MWNTs embedded in an elastic medium are obtained as:

$$
\begin{aligned}
& w_{k}=\sum_{j=1}^{M} A_{k}^{m_{j} n_{j}} \sin \frac{m_{j} \pi x}{L} \cos \left(n_{j} \theta\right) g\left(\omega_{m_{j} n_{j}}, t\right) \\
& (k=1,2, \ldots, N) .
\end{aligned}
$$

Here, the "preferred mode" concept $[15,16,18]$ is used to determine the associated buckling mode, namely that the mode $\left(m_{M}, n_{M}\right)$ associated with the value $\omega=\omega_{\max }=\omega_{m_{M} n_{M}}$ is taken as the corresponding buckling mode.

As a special case, an analytic formula is obtained for dynamic shell buckling of DWNTs embedded in an elastic medium:

$$
\frac{m^{2} \pi^{2}}{\rho L^{2}} \sigma_{x}=\omega^{2}+\frac{1}{2}\left[\alpha+\beta-\sqrt{(\alpha-\beta)^{2}+\gamma^{2}}\right],
$$

where

$$
\begin{aligned}
& \alpha=\frac{1}{\rho h}\left(D \beta_{1}^{2}+c+\frac{m^{4} \pi^{4} E h}{L^{4} R_{1}^{2} \beta_{1}^{2}}\right), \\
& \beta=\frac{1}{\rho h}\left(D \beta_{2}^{2}+c \frac{R_{1}}{R_{2}}+d+\frac{m^{4} \pi^{4} E h}{L^{4} R_{2}^{2} \beta_{2}^{2}}\right), \\
& \gamma=\frac{2 c}{\rho h} \sqrt{\frac{R_{1}}{R_{2}}} .
\end{aligned}
$$

Eq. (19) shows the effect of the van der Waals forces and the surrounding elastic medium. When $\omega=0$, it degenerates to the buckling stress for static case [9]. It can be shown that the static critical buckling stress of DWNTs embedded in an elastic medium increases with increasing the elastic constant $d$.

\section{Numerical results and discussion}

Figure 2 shows the dependence of the critical buckling stress on length-to-outer tube radius ratio for static column buckling and static shell buckling of individual DWNTs at $R_{1}=1,2$ and $4 \mathrm{~nm}$. It can be seen that, for static column buckling, the critical buckling stress is dependent on the aspect ratio and decreases with increasing the aspect ratio. While for static shell buckling, the critical buckling stress is insensitive to the aspect ratio, that is, the effect of aspect ratio on the critical buckling stress can be negligible. Figure 
2 indicates that there exists a critical length-to-outer tube radius ratio (which is about 11.2 for $R_{1}=1 \mathrm{~nm}$ and 15.6 for $R_{1}=2 \mathrm{~nm}$ ) for static shell buckling of individual DWNTs and it increases with increasing the radii of DWNTs.

A similar plot, but for DWNTs embedded in an infinite elastic medium, is given in Figure 3. As that shown in Figure 2 , the critical buckling stress for static column buckling of DWNTs embedded in an elastic medium is dependent on the aspect ratio, while the critical buckling stress for static shell buckling is insensitive to the aspect ratio. However, because of the effect of surrounding elastic medium, the critical buckling stress for static column buckling of DWNTs embedded in an elastic medium decreases first, and then varies near a certain value with increasing the aspect ratio. This differs from the static column buckling of individual DWNTs.

Figures 2 and 3 show that, similar to static buckling of a

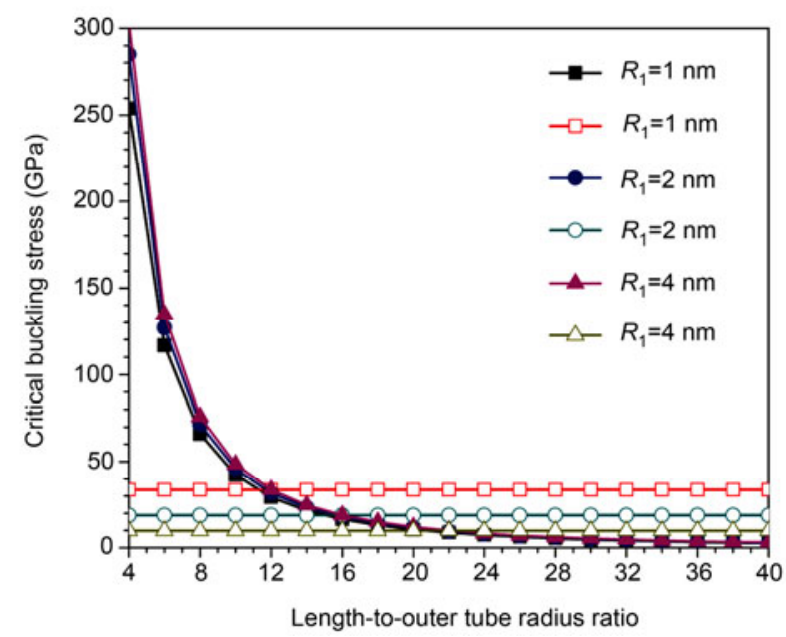

Figure 2 (Color online) Dependence of critical buckling stress on length-to-outer tube radius ratio for static column buckling (solid symbol) and static shell buckling (hollow symbol) of individual DWNTs.

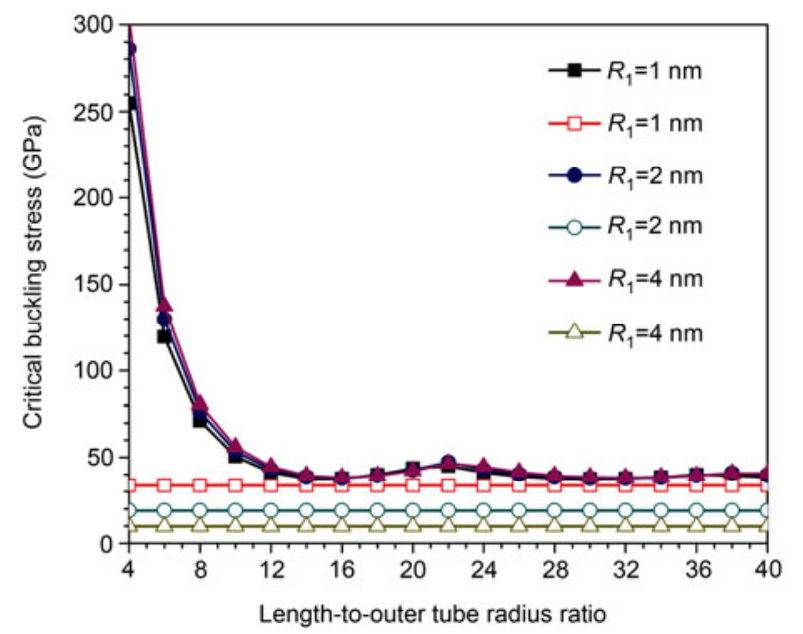

Figure 3 (Color online) Dependence of critical buckling stress on lengthto-outer tube radius ratio for static column buckling (solid symbol) and static shell buckling (hollow symbol) of DWNTs embedded in an infinite elastic medium. cylindrical shell, the column buckling behavior of MWNTs or MWNTs embedded in an elastic medium may occur first at large aspect ratios, so the relative small aspect ratios should be considered for the shell buckling behavior of MWNTs or MWNTs embedded in an elastic medium. Additionally, it is seen from Figures 2 and 3 that the effect of radii on static shell buckling is greater than it on static column buckling for both individual DWNTs and DWNTs embedded in an elastic medium. A comparison of Figure 3 with Figure 2 indicates that, the effect of surrounding elastic medium on the critical buckling stress for static column buckling is greater than that for static shell buckling.

For the model of static column buckling of MWNTs and MWNTs embedded in an elastic medium, refer elsewhere $[16,19]$. In the calculation, the intertube van der Waals interaction coefficient $c^{\prime}$ for the column buckling is taken as $c^{\prime}=320 \times(2 R) \mathrm{erg} / \mathrm{cm}^{2} /\left(0.16 \mathrm{~s}^{2}\right)$, where $R$ denotes the inner tube radius of DWNTs or DWNTs embedded in an elastic medium, $s=0.142 \mathrm{~nm}$. The pressure exerted on the outer tube of DWNTs caused by the surrounding elastic medium $p_{d}^{\prime}$ is described as $p_{d}^{\prime}=-2 d w_{2}$, where $d$ is a spring constant determined by the material properties of both the elastic medium and the outer tube of DWNTs as described by eq. (4).

Tables $1-4$ show the buckling wave number $(m, n)$ on the buckling stress $\sigma_{x}$ for individual DWNTs and DWNTs embedded in an infinite elastic medium with $R_{1}=2 \mathrm{~nm}$ and $R_{1}=4 \mathrm{~nm}$ at $L / R_{2}=8$, respectively. It is seen from Tables $1-4$ that, for individual DWNTs, the buckling form is related to the buckling stress and there exists a critical value which is a little higher than the static critical buckling example. When the buckling stress $\sigma_{x}$ is lower than the critical value, non-axisymmetric buckling occurs, or else axisymmetric buckling occurs. For the DWNTs embedded in an elastic medium, the non-axisymmetric buckling may not take place even for the static buckling because of the effect of surrounding elastic medium. This indicates that DWNTs or embedded DWNTs in dynamic buckling are more susceptible to axisymmetric buckling than non-axisymmetric buckling. The calculations are also performed for individual DWNTs at $\sigma_{x} / \sigma_{c r}=1.1$ and 1.5 and for DWNTs embedded in an infinite elastic medium at $\sigma_{x} / \sigma_{c r}^{d}=1.1$ and 1.5 at aspect ratios $L / R_{2}=5$ and 12 when the inner tube radius ranging from $2 \mathrm{~nm}$ to $30 \mathrm{~nm}$, respectively, where $\sigma_{c r}$ denotes the critical buckling load for static buckling of individual DWNTs and $\sigma_{c r}^{d}$ denotes the one of DWNTs embedded in an infinite elastic medium under otherwise identical conditions. The same results are obtained. Further, for both individual DWNTs and DWNTs embedded in an infinite elastic medium, the axial buckling form shifts from the lower buckling mode to the higher buckling mode with the increase of buckling stress, but the buckling mode is invariable for a certain domain of the buckling stress. A comparison of Tables 3 and 4 with Tables 1 and 2 indicates that, for 
Table 1 Buckling mode $(m, n)$ on buckling stress $\sigma_{x}$ for a DWNT with $R_{1}=2 \mathrm{~nm}$

\begin{tabular}{ccccc}
\hline$(m, n)$ & $(24,4)$ & $(28,2)$ & $(29,0)$ & $(30,0)$ \\
\hline$\sigma_{x}(\mathrm{GPa})$ & $18.906 \leqslant \sigma_{x}<18.971$ & $18.971 \leqslant \sigma_{x}<19.059$ & $19.059 \leqslant \sigma_{x}<19.618$ & $19.618 \leqslant \sigma_{x}<20.971$ \\
\hline \hline$(m, n)$ & $(31,0)$ & $(32,0)$ & $(33,0)$ & $(34,0)$ \\
$\sigma_{x}(\mathrm{GPa})$ & $20.971 \leqslant \sigma_{x}<22.382$ & $22.382 \leqslant \sigma_{x}<23.824$ & $23.824 \leqslant \sigma_{x}<25.294$ & $25.294 \leqslant \sigma_{x}<26.824$ \\
\hline
\end{tabular}

Table 2 Buckling mode $(m, n)$ on buckling stress $\sigma_{x}$ for a DWNT with $R_{1}=4 \mathrm{~nm}$

\begin{tabular}{ccccc}
\hline$(m, n)$ & $(31,6)$ & $(34,5)$ & $(37,3)$ & $(38,2)$ \\
\hline$\sigma_{x}(\mathrm{GPa})$ & $9.870 \leqslant \sigma_{x}<9.879$ & $9.879 \leqslant \sigma_{x}<9.882$ & $9.882 \leqslant \sigma_{x}<9.897$ & $(41,0)$ \\
\hline \hline$(m, n)$ & $(39,0)$ & $(40,0)$ & $(42,0)$ & $10.897 \leqslant \sigma_{x}<9.903$ \\
$\sigma_{x}(\mathrm{GPa})$ & $9.903 \leqslant \sigma_{x}<10.235$ & $10.235 \leqslant \sigma_{x}<10.765$ & $10.765 \leqslant \sigma_{x}<11.294$ & $11.294 \leqslant \sigma_{x}<11.853$ \\
\hline
\end{tabular}

Table 3 Buckling mode $(m, n)$ on buckling stress $\sigma_{x}$ for a DWNT embedded in an infinite elastic medium with $R_{1}=2 \mathrm{~nm}$

\begin{tabular}{|c|c|c|c|c|}
\hline$(m, n)$ & $(28,2)$ & $(29,0)$ & $(30,0)$ & $(31,0)$ \\
\hline$\sigma_{x}(\mathrm{GPa})$ & $18.972 \leqslant \sigma_{x}<19.059$ & $19.059 \leqslant \sigma_{x}<19.618$ & $19.618 \leqslant \sigma_{x}<20.971$ & $20.971 \leqslant \sigma_{x}<22.382$ \\
\hline$(m, n)$ & $(32,0)$ & $(33,0)$ & $(34,0)$ & $(35,0)$ \\
\hline
\end{tabular}

Table 4 Buckling mode $(m, n)$ on buckling stress $\sigma_{x}$ for a DWNT embedded in an infinite elastic medium with $R_{1}=4 \mathrm{~nm}$

\begin{tabular}{ccccc}
\hline$(m, n)$ & $(39,0)$ & $(40,0)$ & $(41,0)$ & $(42,0)$ \\
\hline$\sigma_{x}(\mathrm{GPa})$ & $9.920 \leqslant \sigma_{x}<10.235$ & $10.235 \leqslant \sigma_{x}<10.765$ & $10.765 \leqslant \sigma_{x}<11.294$ & $(45,0)$ \\
\hline \hline$(m, n)$ & $(43,0)$ & $(44,0)$ & $(46,0)$ \\
$\sigma_{x}(\mathrm{GPa})$ & $11.853 \leqslant \sigma_{x}<12.412$ & $12.412 \leqslant \sigma_{x}<13.000$ & $13.000 \leqslant \sigma_{x}<13.588$ & $13.588 \leqslant \sigma_{x}<14.176$ \\
\hline
\end{tabular}

dynamic shell buckling of DWNTs embedded in an elastic medium, the lowest buckling stress is enhanced by the surrounding elastic medium when compared with the corresponding one for individual DWNTs. The surrounding elastic medium only has influence on the lower axial buckling mode.

Figures 4 and 5 show the dependence of the axial buckling wavelength on inner tube radius for individual DWNTs and DWNTs embedded in an infinite elastic medium, respectively. It can be seen from Figures 4 and 5 that, for both individual DWNTs and DWNTs embedded in an infinite elastic medium, the axial buckling wavelength increases with increasing the inner tube radius and the lower buckling stress corresponds to a larger buckling wavelength.

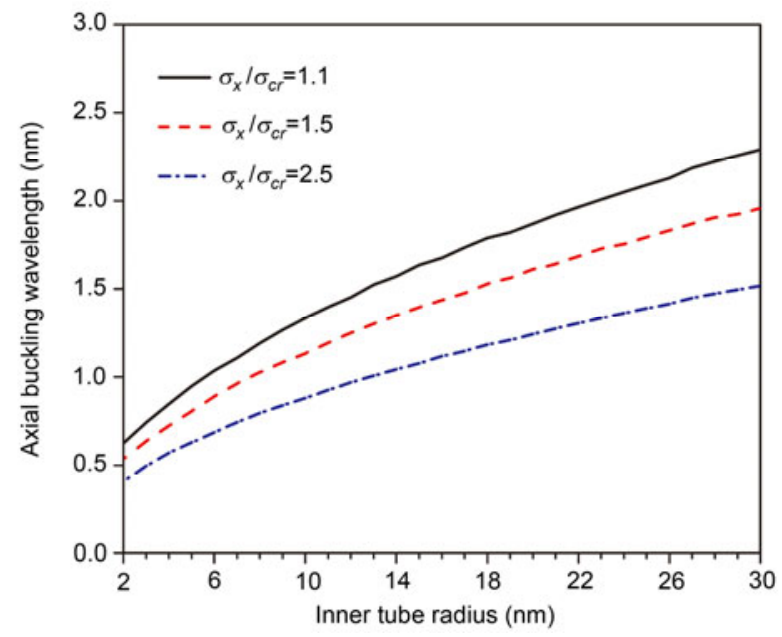

Figure 4 (Color online) Axial buckling wavelength on inner tube radius for individual DWNTs at $\sigma_{x} / \sigma_{c r}=1.1,1.5$ and 2.5 .

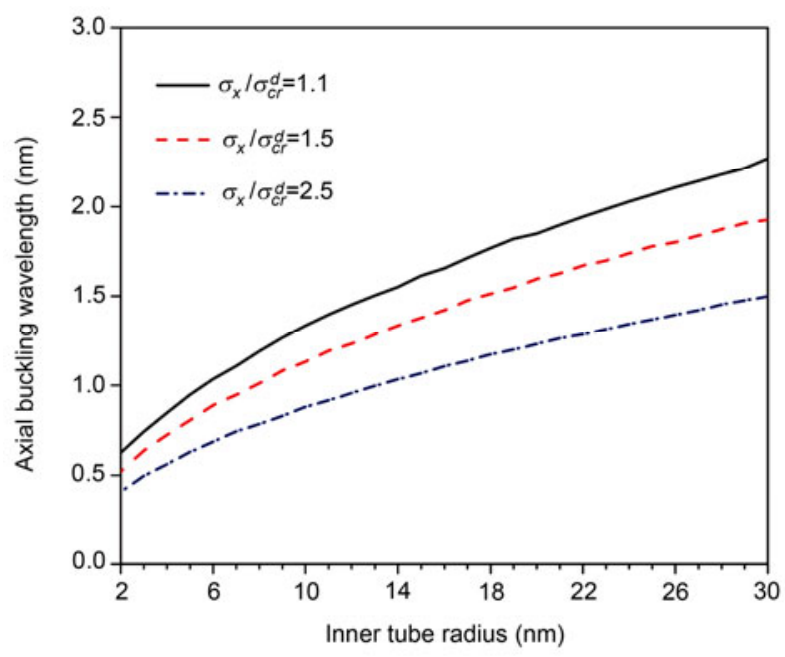

Figure 5 (Color online) Axial buckling wavelength on inner tube radius for DWNTs embedded in an infinite elastic medium at $\sigma_{x} / \sigma_{c r}^{d}=1.1,1.5$ and 2.5.

\section{Approximate method for dynamic axisym- metric buckling of embedded DWNTs}

It has been shown in sect. 4 that DWNTs or DWNTs embedded in an elastic medium in dynamic shell buckling are prone to axisymmetric buckling rather than non-axisymmetric buckling. When $n=0$, eq. (19) degenerates to the case for dynamic axisymmetric buckling of DWNTs embedded in an elastic medium

$$
\sigma_{a x}=D \frac{m^{2} \pi^{2}}{h L^{2}}+\frac{L^{2}}{h m^{2} \pi^{2}} X,
$$


where

$$
\begin{aligned}
X= & \frac{1}{2}\left(2 \rho h \omega^{2}+d+c+c \frac{R_{1}}{R_{2}}+\frac{E h}{R_{1}^{2}}+\frac{E h}{R_{2}^{2}}\right) \\
& -\frac{1}{2} \sqrt{\left(d+\frac{E h}{R_{2}^{2}}+\frac{c R_{1}}{R_{2}}-\frac{E h}{R_{1}^{2}}-c\right)^{2}+4 \frac{c^{2} R_{1}}{R_{2}} .}
\end{aligned}
$$

Assume that the length of a DWN, $L$, is larger than its radii, the minimum value of $\sigma_{a x}$ can be approximated by

$$
\sigma_{a x}=2 \sqrt{D X} / h
$$

with the corresponding axial buckling wavelength as:

$$
\lambda=\frac{L}{m}=\pi \sqrt[4]{\frac{D}{X}}=\pi \sqrt{\frac{2 D}{\sigma_{a x} h}} .
$$

Eqs. (21) and (22) have the same forms as the expressions for the static critical axial stress and the associated axial wavelength for axisymmetric buckling of a cylindrical shell [20]. Eq. (22) indicates that the axial buckling wavelength is independent of the length of DWNTs and decreases with increasing the applied axial stress.

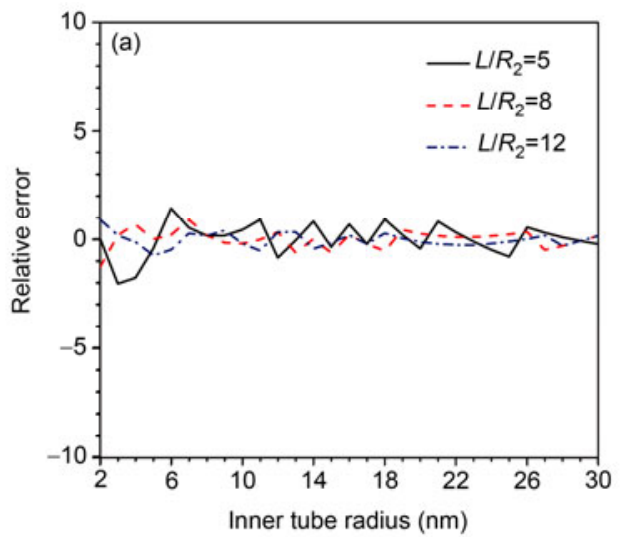

In the following, it will be shown that eq. (22) can well approximate the relation of the buckling stress and the associated buckling wavelength for dynamic axisymmetric buckling of DWNTs or embedded DWNTs, even for DWNTs or embedded DWNTs with small aspect ratios.

Figure 6 displays the relative error of buckling wavelength $\lambda$ of individual DWNTs obtained by eq. (22) over that by eq. (20) for different aspect ratios $L / R_{2}=5,8$ and 12 at the stress ratios $\sigma_{x} / \sigma_{c r}=1.1$ and 1.5. It is seen that, with a wide range of the inner tube radius (from $2 \mathrm{~nm}$ to $30 \mathrm{~nm}$ ), the relative error of $\lambda$ by eq. (22) over that by eq. (20) is very small (less than $2.5 \%$ ) for all the aspect ratios $L / R_{2}=5$, 8 and 12 and the stress ratios $\sigma_{x} / \sigma_{c r}=1.1$ and 1.5.

Figure 7 presents the relative error of buckling wavelength $\lambda$ of DWNTs embedded in an infinite elastic medium obtained by eq. (22) over that by eq. (20) for different aspect ratios $L / R_{2}=5,8$ and 12 at the stress ratios $\sigma_{x} / \sigma_{c r}^{d}=1.1$ and 1.5. Similar to the result for individual DWNTs, the relative error of $\lambda$ by eq. (22) over that by eq. (20) is also very small with a wide range of the inner tube radius (from $2 \mathrm{~nm}$ to $30 \mathrm{~nm}$ ) for all the aspect ratios $L / R_{2}=5,8$ and 12 and the stress ratios $\sigma_{x} / \sigma_{c r}^{d}=1.1$ and 1.5. These results

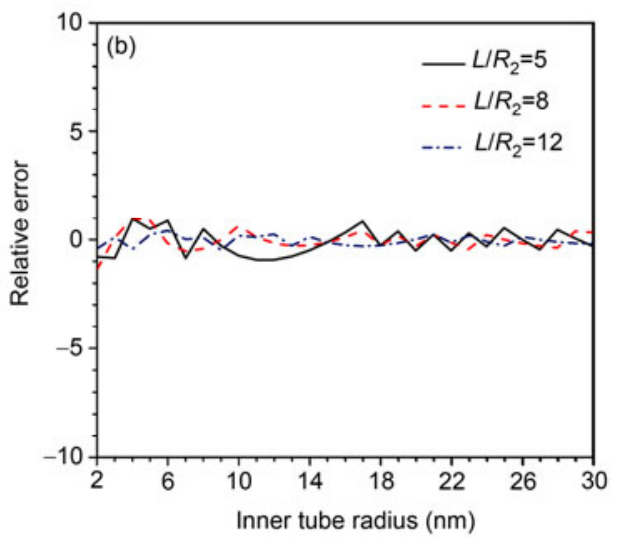

Figure 6 (Color online) Relative error of buckling wavelength obtained by eq. (22) over that by eq. (20) for individual DWNTs. (a) $\sigma_{x} / \sigma_{c r}=1.1$; (b) $\sigma_{x} / \sigma_{c r}=1.5$.
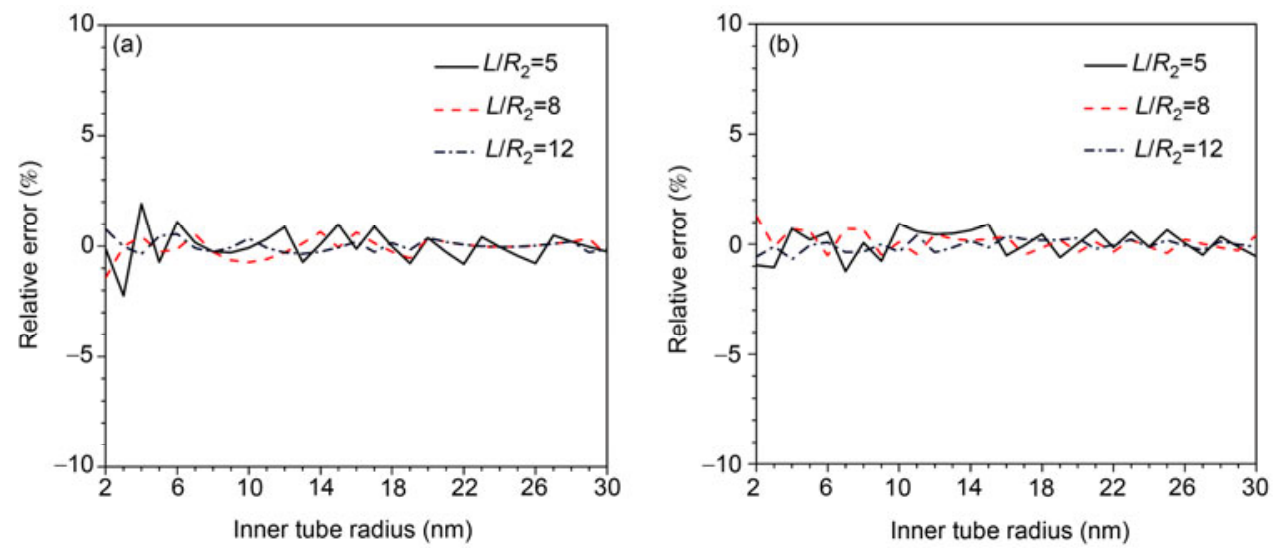

Figure 7 (Color online) Relative error of buckling wavelength obtained by eq. (22) over that by eq. (20) for DWNTs embedded in an infinite elastic medium. (a) $\sigma_{x} / \sigma_{c l}=1.1$; (b) $\sigma_{x} / \sigma_{c r}=1.5$. 
indicate that the analytic formula eq. (22) can well approximate the relation of the buckling stress and the associated buckling wavelength for dynamic axisymmetric buckling of individual DWNTs or DWNTs embedded in an elastic medium.

\section{Conclusions}

This paper studied the dynamic shell buckling behavior of MWNTs embedded in an elastic medium under step axial load based on continuum mechanics model, which takes into account the effect of radial constraint from the surrounding elastic medium and the van der Waals forces between adjacent layers. By introducing initial imperfections of MWNTs and applying the preferred mode analytical method, a buckling condition is derived for the buckling stress and the associated buckling mode. The paper shows that, for occurrence of dynamic shell buckling of MWNTs or MWNTs embedded in an elastic medium, the buckling stress is higher than the critical one for the corresponding static buckling under otherwise identical conditions. In particular, detailed results are demonstrated for dynamic buckling behavior of individual DWNTs and DWNTs embedded in an elastic medium, and analytical formula is obtained. Numerical results indicate that DWNTs or embedded DWNTs in dynamic shell buckling are susceptible to axisymmetric buckling rather than non-axisymmetric buckling. Further, an approximate analytic formula is presented for the relation between the buckling stress and the associated buckling wavelength for dynamic axisymmetric buckling of embedded DWNTs. The present investigation may be useful in understanding the mechanical property of MWNTs and their potential applications.

This work was supported by the National Natural Science Foundation of China (Grant Nos. 11172304, 11202210, 11021262 and 10972010) and the National Basic Research Program of China (Grant No. 2012CB937500).

1 Thostenson E T, Ren Z, Chou T W. Advances in the science and technology of carbon nanotubes and their composites: A review.
Compos Sci Technol, 2001, 61: 1899-1912

2 Dharap P, Li Z L, Nagarajaiah S, et al. Nanotube film based on single-wall carbon nanotubes for strain sensing. Nanotechnology, 2004, 15: $379-382$

3 Waters J F, Guduru P R, Jouzi M, et al. Shell buckling of individual multiwalled carbon nanotubes using nanoindentation. Appl Phys Lett, 2005, 87: 103109

4 Wang X, Lu G, Lu Y J. Buckling of embedded multi-walled carbon nanotubes under combined torsion and axial loading. Int J Solids Struct, 2007, 44: 336-351

5 Sun Y G, Yao X H, Han Q. Combined torsional buckling of doublewalled carbon nanotubes with axial load in the multi-field coupled condition. Sci China-Phys Mech Astron, 2011, 54: 1659-1665

6 Miao C Y, Li H J, Guo W L. Radial breathing modes of multi-walled carbon nanotubes by an atomic beam-spring model. Sci China-Phys Mech Astron, 2012, 55: 940-946

7 Yakobson B I, Brabec C J, Bernholc J. Nanomechanics of carbon tubes: Instability beyond linear response. Phys Rev Lett, 1996, 76: 2511-2514

8 Ru C Q. Effect of van der Waals forces on axial buckling of a double-walled carbon nanotube. J Appl Phys, 2000, 87: 7227-7231

9 Ru C Q. Axially compressed buckling of a doublewalled carbon nanotube embedded in an elastic medium. J Mech Phys Solids, 2001, 49: 1265-1279

10 Wang L F, Zheng Q S, Liu J Z, et al. Size dependence of the thinshell model for carbon nanotubes. Phys Rev Lett, 2005, 95: 105501

11 Wang C Y, Ru C Q, Mioduchowski A. Axially compressed buckling of pressured multiwall carbon nanotubes. Int J Solids Struct, 2003, 40: 3893-3911

12 Wang C Y, Ru C Q, Mioduchowski A. Applicability and limitations of simplified elastic shell equations for carbon nanotubes. ASME J Appl Mech, 2004, 71: 622-631

13 Peng J, Wu J, Hwang K C, et al. Can a single-wall carbon nanotube be modeled as a thin shell? J Mech Phys Solids, 2008, 56: 2213-2224

14 Ajayan P M, Zhou O Z. Applications of carbon nanotubes. Top Appl Phys, 2001, 80: 391-425

15 Sun C, Liu K, Lu G. Dynamic torsional buckling of multi-walled carbon nanotubes embedded in an elastic medium. Acta Mech Sin, 2008, 24: 541-547

16 Sun C, Liu K. Dynamic column buckling of multi-walled carbon nanotubes under axial impact load. Solid State Commun, 2009, 149: 429-433

17 Zhang Y C, Wang X. Thermal effects on interfacial stress transfer characteristics of carbon nanotubes/polymer composites. Int J Solids Struct, 2005, 42: 5399-5412

18 Lindberg H E, Herbert R E. Dynamic buckling of a thin cylindrical shell under axial impact. ASME J Appl Mech, 1966, 32(3): 105-112

19 Ru C Q. Column buckling of multiwalled carbon nanotubes with interlayer radial displacements. Phys Rev B, 2000, 62: 16962-16967

20 Timoshenko S P, Gere J M. Theory of Elastic Stability. 2nd ed. New York: McGraw-Hill Book Company, Inc., 1961. 457-459 